\title{
Nanoethosomal transdermal delivery of vardenafil for treatment of erectile dysfunction: optimization, characterization, and in vivo evaluation
}

This article was published in the following Dove Press journal:

Drug Design, Development and Therapy

18 November 2015

Number of times this article has been viewed

\section{Usama A Fahmy}

Department of Pharmaceutics \& Industrial Pharmacy, Faculty of Pharmacy, King Abdulaziz University, Jeddah, Saudi Arabia
Correspondence: Usama A Fahmy Department of Pharmaceutics \& Industrial Pharmacy, Faculty of Pharmacy, King Abdulaziz University, PO Box 80200, 21589 Jeddah, Saudi Arabia Email usamafahmy@hotmail.com

\begin{abstract}
Vesicular drug delivery systems have recently gained attention as a way of improving dosing accuracy for drugs with poor transdermal permeation. The current study focuses on utilization of the natural biocompatible vesicles to formulate vardenafil nanoethosomes (VRD-NE), for the enhancement of their transdermal permeation and bioavailability. Fifteen formulations were prepared by thin-layer evaporation technique according to Box-Behnken design to optimize formulation variables. The effects of lipid composition, sonication time, and ethanol concentration on particle size and encapsulation efficiency were studied. The diffusion of vardenafil (VRD) from the prepared nanoethosomes specified by the design was carried out using automated Franz diffusion cell apparatus. The optimized formula was investigated for in vivo pharmacokinetic parameters compared with oral VRD suspension. Confocal laser scanning microscopy images were used to confirm enhanced diffusion release of VRD in rat skin. The results showed that the optimized formula produced nanoethosomes with an average size of $128 \mathrm{~nm}$ and an entrapment efficiency of $76.23 \%$. VRD-NE provided a significant improvement in permeation with an enhancement ratio of 3.05-fold for a film made with optimally formulated VRD-NE compared with a film made with VRD powder. The transdermal bioavailability of VRD from the nanoethosome film was approximately twofold higher than the oral bioavailability from an aqueous suspension. VRD-NE thus provide a promising transdermal drug delivery system. As a result, management of impotence for a longer duration could be achieved with a reduced dosage rate that improves patient tolerability and compliance for the treatment of erectile dysfunction.
\end{abstract}

Keywords: Box-Behnken design, impotence, vesicles, nanoparticles

\section{Introduction}

Erectile dysfunction (ED), the inability to develop or maintain sufficient erection, is widespread globally, and in developed countries. ${ }^{1}$ Recent studies have shown that the prevalence of ED is $>40 \%$ in Arab countries; this high prevalence is linked to several risk factors, including aging, obesity, lack of exercise, smoking, and the complications of diabeties mellitus. ${ }^{2}$ Vardenafil (VRD), a 5-phosphodiesterase (PDE5) inhibitor that blocks the enzyme in the smooth muscle cells that line the blood vessels supplying the corpus cavernosum of the penis, is one of the drugs of choice for the treatment of ED. Inhibition of PDE5 relaxes the blood vessels and increases the blood flow, resulting in an erection in response to sexual stimulation. ${ }^{3}$ Although VRD has low oral bioavailability (15\%), it has significant advantages compared with sildenafil and tadalafil, the most commonly used alternatives for the treatment of ED. VRD is a more selective inhibitor of PDE5 than tadalafil or sildenafil and is ten times more potent than sildenafil. ${ }^{4-6}$ VRD is thus used at a lower dose than tadalafil or sildenafil to maintain 
penile erection, potentially resulting in fewer side effects as runny or stuffy nose, headache, dizziness, upset stomach, and back pain. The main reasons for the low oral bioavailability of VRD are extensive metabolism and low solubility; VRD is designated a class II drug according to Biopharmaceutical Classification System. ${ }^{7,8}$

The use of lipid vesicles as transdermal drug delivery systems has attracted increasing attention in recent years. ${ }^{9}$ It is generally accepted, however, that conventional liposomes would be unsuitable for transdermal delivery of VRD, since drugs delivered in this way remain confined to the upper layer of stratum corneum. ${ }^{10}$

The discovery of ethosomes by Touitou et al widened the possibilities for using lipid vesicles for transdermal drug delivery. ${ }^{11}$ Ethosomes are lipid vesicles incorporating relatively high concentrations of ethanol that were designed to deliver drugs across the skin layers. Although ethosomes show significantly higher transdermal flux compared with liposomes and transfersomes, the exact reasons for this remain unclear; the enhanced permeation into deeper skin layers and distribution into lipid bilayers have been postulated to result from the synergistic effects of phospholipids and a high ethanol concentration in the vesicules. ${ }^{12}$ In a previous study, in vitro skin permeation of an ethosomal formulation of zidovudine was shown to be $\sim 24$-fold higher than that of an aqueous solution of the drug. ${ }^{13}$

Few attempts have been made to enhance the oral bioavailability of VRD; one of these is the development of an orodispersible tablet formulation. ${ }^{14}$ There remains, however, a need to enhance absorption and overcome the short duration of action of VRD. The aim of the present study was to improve the bioavailability of VRD by enhancing its solubility and to increase its duration of action. It was hoped that this could be achieved by incorporation of VRD into a nanosized lipid vesicular matrix. Application as a transdermal film would avoid first-pass metabolism in the liver and lower the dose needed to maintain an erection, potentially reducing side effects.

\section{Materials and methods \\ Materials}

VRD was purchased from Jinlan Pharm-Drugs Technology Co., Ltd. (Hangzhou, People's Republic of China). L- $\alpha$-Phosphatidylcholine (soya 95\%) was purchased from Avanti Polar Lipids (Alabaster, AL, USA). Hydroxypropyl methylcellulose (HPMC) and cholesterol (CHO) were purchased from Sigma-Aldrich Chemical Company (St Louis, MO, USA). Potassium dihydrogen phosphate was procured from BDH Chemicals Ltd. (Poole, UK). All other chemicals and reagents used were of analytical grade.

\section{Methodology}

\section{Formulation of VRD nanoethosomes}

VRD nanoethosomes (VRD-NE) were formulated using a thin-layer evaporation technique. ${ }^{15}$ Fifteen sets of experimental conditions were constructed using Statgraphics Plus, Version 4 (Manugistics Inc., Rockville, MD, USA), with a BoxBehnken factorial design. The independent variables were the following: \% L- $\alpha$-phosphatidylcholine $\left(X_{1}\right)$ to total lipids, $\mathrm{L}-\alpha-$ phosphatidylcholine, and $\mathrm{CHO}$; ultrasonication time in minutes $\left(X_{2}\right)$; and \% ethanol in the hydration medium $\left(X_{3}\right)$. These preliminary studies provided a basis for selecting the levels of each variable in the formulation. The selected responses were mean particle size $\left(Y_{1}\right)$ and VRD encapsulation efficiency (EE) $\left(Y_{2}\right)$. Variables and measured responses of the design experiment are presented in Table 1 . The total lipid concentration was 3\% $(w / v)$ of the total weight of each formulation.

L- $\alpha$-Phosphatidylcholine and drug were weighed in a round-bottom flask and dissolved in chloroform-methanol $(1: 1, \mathrm{v} / \mathrm{v})$. The organic solvent was evaporated using a rotary evaporator (Büchi Rotavapor R-200; Büchi Labortechnik AG, Flawi, Switzerland), and traces of residual solvent were removed in a vacuum oven (Model 6505; Thermo Fisher Scientific, Waltham, MA, USA) overnight. The precipitated lipid film was resuspended in ethanol-water using different ethanol ratios (Table 2). The produced vesicles were kept at room temperature for 2 hours to encapsulate the VRD and then ultrasonicated using a probe sonicator (VC750; Sonics, Newtown, CT, USA) at $2^{\circ} \mathrm{C}$ for different lengths of time.

\section{Formulation of optimized (VRD-NE) transdermal films}

Based upon the previous trials for film formation, HPMC $(1.5 \%, \mathrm{w} / \mathrm{v})$ polymers were used as the film matrix to which the optimized formulation was added. The solution was stirred with a magnetic stirrer for 2 hours and then poured

Table I Independent and dependent variables and their levels for Box-Behnken design

\begin{tabular}{|c|c|c|c|c|c|c|c|}
\hline \multirow[t]{2}{*}{ Factors } & \multicolumn{3}{|c|}{ Levels } & \multicolumn{4}{|c|}{ Constraints } \\
\hline & -1 & 0 & $+I$ & Response & Minimum & Maximum & Goal \\
\hline$X_{1}$ & 10 & 50 & 90 & $Y_{1}$ & 98 & 543 & Minimize \\
\hline$X_{2}$ & 1 & 3 & 5 & & & & \\
\hline$X_{3}$ & 30 & 55 & 80 & $Y_{2}$ & 10.12 & 78.76 & Maximize \\
\hline
\end{tabular}

Abbreviations: $X_{1}$, \% L- $\alpha$-phosphatidylcholine to total lipids; $X_{2}$, ultrasonication time (minutes); $X_{3}$, \% ethanol in the hydration medium; $Y_{1}$, mean particle size; $Y_{2}$, vardenafil encapsulation efficiency. 
Table 2 Experimental runs and their observed responses

\begin{tabular}{|c|c|c|c|c|c|}
\hline \multirow[t]{2}{*}{ Run } & \multicolumn{3}{|c|}{ Factors } & \multicolumn{2}{|c|}{ Responses } \\
\hline & $x_{1}$ & $x_{2}$ & $x_{3}$ & $Y_{1}^{*}$ & $Y_{2}^{*}$ \\
\hline $\mathrm{I}$ & 90 & 3 & 30 & 213 & 78.76 \\
\hline 2 & 10 & 5 & 55 & 324 & 26.34 \\
\hline 3 & 10 & 3 & 30 & 432 & 27.53 \\
\hline 4 & 90 & 5 & 55 & 132 & 55.34 \\
\hline 5 & 50 & 3 & 55 & 231 & 43.34 \\
\hline 6 & 50 & 5 & 80 & 98 & 13.42 \\
\hline 7 & 90 & 3 & 80 & 176 & 40.32 \\
\hline 8 & 10 & 3 & 80 & 287 & 10.12 \\
\hline 9 & 50 & 3 & 55 & 178 & 21.93 \\
\hline 10 & 10 & 1 & 55 & 165 & 23.54 \\
\hline 11 & 90 & 1 & 55 & $54 I$ & 12.01 \\
\hline 12 & 50 & 3 & 55 & 189 & 36.42 \\
\hline 13 & 50 & 1 & 30 & 543 & 52.53 \\
\hline 14 & 50 & 5 & 30 & 145 & 53.76 \\
\hline 15 & 50 & I & 80 & 435 & 23.21 \\
\hline
\end{tabular}

Note: $*$ SD values were $<5 \%$.

Abbreviations: SD, standard deviation; $X_{1}, \% \mathrm{~L}-\alpha$-phosphatidylcholine to total lipids; $X_{2}$, ultrasonication time (minutes); $X_{3}$, \% ethanol in the hydration medium; $Y_{1}$, mean particle size; $Y_{2}$, vardenafil encapsulation efficiency.

into a petri dish and maintained at $40^{\circ} \mathrm{C}$ in an oven until the solvent was completely evaporated. Two films are prepared, one using unprocessed VRD and the other using VRD-NE.

\section{Characterization of VRD-NE}

\section{Vesicle structural and surface morphology}

A transmission electron microscope (CM12 TEM; Philips, Amsterdam, the Netherlands) was used to determine VRD-NE morphology. The optimized formulation was stained with an aqueous solution of phosphotungstic acid ( $1 \%, \mathrm{w} / \mathrm{v})$ prior to use. The sample was examined using an accelerated voltage of $20 \mathrm{kV}$, with a magnification of $\times 4,000$.

\section{Vesicle size analysis}

The vesicle size of the optimized VRD-NE formulation was determined using Zetatrac (Microtrac, Montgomeryville, PA, USA) at $20^{\circ} \mathrm{C}$. Each measurement was carried out in triplicate.

\section{Entrapment efficiency}

Free VRD was determined indirectly by centrifugation at $20,000 \mathrm{rpm}$ for 45 minutes at $8^{\circ} \mathrm{C}$ using a cooling centrifuge (laboratory centrifuge K30, Sigma, Osterode, Germany). An aliquot of the supernatant $(500 \mu \mathrm{L})$ was diluted with aqueous $\mathrm{HCl}(0.1 \mathrm{~N})$ and injected directly into a high-performance liquid chromatography (HPLC) instrument using the method described by Carlucci et al, ${ }^{16}$ with measurement at $230 \mathrm{~nm}$. VRD EE was determined by Equation 1.

Encapsulation
efficiency $(w / w, \%)=\frac{\text { Amount of VRDin the nano ethosomes }}{\text { Weight of VRDinitially added }}(1)$

\section{Ex vivo skin permeation studies}

A Franz diffusion cell apparatus (Microette Plus; Hanson Research, Chatsworth, CA, USA) was used to assess the diffusion of prepared films. Skin ( $2 \mathrm{~cm}^{2}$ used as a membrane) was obtained from the abdominal region of male rats after hair removal. Each diffusion cell had a donor and receptor chamber, with a film placed between them. The receptor medium was phosphate-buffered saline ( $\mathrm{pH} 7.2)$, the stirring rate was $400 \mathrm{rpm}$, and the temperature was maintained at $32^{\circ} \mathrm{C} \pm 0.5^{\circ} \mathrm{C}$. The autosampler collected aliquots at $0.5,1,2$, $4,8,12,24$, and 36 hours, and these were then analyzed by HPLC. Skin permeation parameters, steady-state transdermal flux $\left(J_{\mathrm{ss}}\right)$, diffusion coefficient $(D)$, enhancement ratio (ER), and permeability coefficient $\left(K_{\mathrm{p}}\right)$, were calculated from the in vitro skin permeation data. The cumulative amount of VRD permeated per unit area of skin was plotted as a function of time. $J_{\text {ss }}$ and lag time were calculated from the slope and $X$-intercept of the linear portion of the graph, respectively. ER was calculated by dividing $J_{\text {ss }}$ of the optimized ethosomal system by that of the unprocessed drug transdermal film. $K_{\mathrm{p}}$ was calculated using Equation 2.

$$
K_{\mathrm{p}}=J_{\mathrm{ss}} / C
$$

$D$ was calculated using Equation 3:

$$
T_{\mathrm{L}}=h^{2} / 6 D
$$

where $h$ is skin thickness, $T_{\mathrm{L}}$ is lag time, $C$ is film initial concentration, and $D$ is diffusion coefficient.

\section{Confocal laser scanning microscopy}

For confocal laser scanning microscopy (CLSM), a fluorescent dye, Rhodamine B (Rh), with a concentration of $0.1 \%$ $(\mathrm{w} / \mathrm{v})$ was incorporated in the optimized VRD-NE. The fluorescent nanoethosomes were poured into the HPMC solution as described previously to form VRD-NE-HPMC film. Another HPMC film was formed with plain dye. The films were applied to the skin pieces. The procedure used for ex vivo permeation study was similar to that prescribed in the "Ex vivo skin permeation studies" section. After 1 and 4 hours, the skin was removed from the diffusion cells, rinsed with distilled water, and preserved in $10 \%(\mathrm{v} / \mathrm{v})$ formalin in phosphate-buffered saline ( $\mathrm{pH} 7.4$ ) for 24 hours. The skin was vertically cross-sectioned into pieces of $0.5 \mathrm{~mm}^{2}$ size and evaluated for probe penetration. The full skin thickness was optically scanned. Visualization was performed by the $\times 10$ objective lens system of an inverted Zeiss LSM 510 META 
microscope (Carl Zeiss, Jena, Germany), equipped with a He-Ne laser. The excitation wavelength used was $562 \mathrm{~nm}$, while emission wavelength was $587 \mathrm{~nm} .{ }^{17}$

\section{Bioavailability study}

All experimental protocols were approved by the Animal Ethics Committee of King Abdulaziz University, Jeddah, Saudi Arabia, and conformed to the Declaration of Helsinki and the Guiding Principle in Care and Use of Animals (DHEW publication NIH 80-23) and the "Principles of Laboratory Animal Care" (NIH publication \#85-23, revised in 1985). Male Wistar rats $(350 \pm 50 \mathrm{~g})$ were used for the pharmacokinetic study. The rats were acclimated for at least 7 days in environmentally controlled cages $\left(23^{\circ} \mathrm{C} \pm 1^{\circ} \mathrm{C}\right.$ and $12 / 12$ hour dark/light cycle) with free access to standard food and water. The rats were fasted overnight before the experiments. The animals were divided into two groups (four rats per group). The first group received an oral dose of VRD $(2 \mathrm{mg} / \mathrm{kg})$ suspended in water $(1 \mathrm{~mL})$. In the second group, a VRD-NE film was applied to a shaved patch on the back of the animal at a dose equivalent to $2 \mathrm{mg} / \mathrm{kg}$ VRD. Two hundred microliters of blood from each rat was withdrawn at $0,1,2,4,8,12,24$, and 36 hours.

Pharmacokinetic parameters based on VRD plasma concentrations were analyzed with PK-SOLVER software using a noncompartmental model. Maximum plasma concentration $\left(C_{\max }\right)$, time to maximum concentration $\left(T_{\max }\right)$, area under the plasma concentration-time curve (AUC), and mean residency time (MRT) were calculated for individual rats.

\section{Statistical analysis of data}

Data are presented as mean \pm standard deviation. Significant differences between the pharmacokinetic data were tested by analysis of variance followed by Tukey's multiple comparisons test; the confidence level was set at $P<0.05$ (GraphPad Prism 6; GraphPad Software, La Jolla, CA, USA).

\section{Quantification of VRD in plasma by HPLC-DAD detector}

Plasma drug concentrations were analyzed using an Agilent HPLC instrument 1200 (Agilent Technologies, Santa Clara, CA, USA), with a 1200 diode array detector (DAD) detector set at a wavelength of $230 \mathrm{~nm}$. An isocratic mobile phase (acetonitrile:potassium dihydrogen orthophosphate buffer, $10 \mathrm{mM}[1: 1, \mathrm{v} / \mathrm{v}]$, adjusted to $\mathrm{pH} 3$ with orthophosphoric acid) was used for the elution. The separation was carried out using an ODS-A C18 analytical column $(250 \mathrm{~mm} \times 4.6 \mathrm{~mm}$ i.d., ACE Ltd, Zurich Switzerland), with a flow rate of $1.3 \mathrm{~mL} / \mathrm{min}$. The column was maintained at $40^{\circ} \mathrm{C}$, and the injection volume was $20 \mu \mathrm{L}$. The HPLC conditions and VRD plasma extraction procedure were adapted from a method described by Carlucci et al. ${ }^{16}$

\section{Results and discussion}

A Box-Behnken design was used in this study to optimize a nanoethosomal film formulation of VRD. This approach proposed different formulations to enhance the clinical efficacy of VRD in treating ED, while reducing side effects commonly associated with PDE5 inhibitors. By using a BoxBehnken design, each independent variable was investigated at three levels, and the outcome was based on two responses, nanoethosomal vesicular size and EE. Optimized conditions were determined by experimentally testing the 15 formulations generated by the design. Pareto charts (Figure 1) were used to illustrate the effect of the experimental variables on the responses. A positive sign in the chart indicates a direct relationship between the variable and the observed response, whereas a negative sign indicates an inverse relationship.
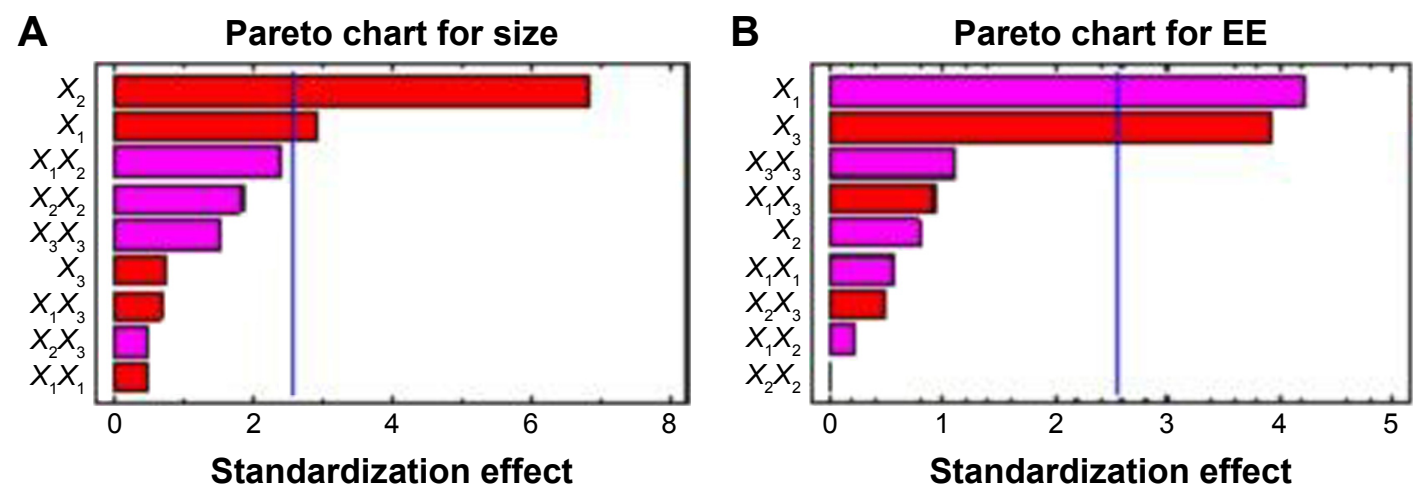

\section{$+\square-$}

Figure I Standard Pareto charts showing the effects of independent variables and their combined effects on particle size (A) and VRD EE (B).

Abbreviations: VRD, vardenafil; EE, encapsulation efficiency; $X_{1}, \% \mathrm{~L}-\alpha$-phosphatidylcholine to total lipids; $X_{2}$, ultrasonication time (minutes); $X_{3}, \%$ ethanol in the hydration medium. 


\section{Vesicle size}

The Pareto chart showed a significant inverse effect of both $L-\alpha$-phosphatidylcholine and sonication time on vesicle size. The interaction of these variables and their quantitative effects on the observed responses were generated using a mathematical regression equation (Equation 4) for the observed response $Y_{1}$.

$$
\begin{aligned}
Y_{1}= & 1,249.37-1.6321 * X_{1}-205.712 * X_{2}-12.2147 * X_{3} \\
& +0.000967708 * X_{1}^{2}+0.10125 * X_{1} * X_{2}+0.0054 * X_{1} * X_{2} \\
& +16.7396 * X_{2}+0.305 * X_{2} * X_{3}+0.0751333 * X_{3}^{2}
\end{aligned}
$$

Several reports have demonstrated the importance of $L-\alpha-$ phosphatidylcholine on vesicular size in the preparation of NE. These reports describe the role of L- $\alpha$-phosphatidylcholine, with a hydrophile-lipophile balance value of 4 , as an emulsifying agent. ${ }^{18}$ The vesicle size of NE decreased with increasing concentration of surfactant. All emulsifying agents concentrate at the aqueous:nonaqueous interface and provide a protective barrier around the dispersed droplets; emulsifiers stabilize the emulsion by reducing the interfacial tension of the system. Some agents enhance the stability by imparting a charge on the droplet surface, thus reducing the physical contact between the droplets and decreasing the potential for coalescence. In our experiments, however, increased amounts of $\mathrm{CHO}$ increased the ethosomal vesicle size, in agreement with data published by Essa. ${ }^{19}$ This effect may be attributed to the amphipathic nature of $\mathrm{CHO}$, which has hydrophilic head (hydroxyl group) and a hydrophobic tail (hydrocarbon chain). When $\mathrm{CHO}$ is incorporated in the bilayer membrane, the hydrophilic head is oriented toward the aqueous phase, and the aliphatic tail lies parallel to the hydrocarbon chains. $\mathrm{CHO}$ is known to increase the chain order of the liquid-state bilayer and strengthen the nonpolar tail of the nonionic surfactant. Accordingly, at low $\mathrm{CHO}$ concentrations, it is expected that $\mathrm{CHO}$ would result in close packing of surfactant monomers, increased curvature, and reduced size of NE vesicles. Conversely, higher concentrations of lipophilic $\mathrm{CHO}(\log P 7.02)$ would reduce the nonionic surfactant content, increase the hydrophobicity, and disturb the vesicular bilayer membrane, thus increasing the size of NE vesicles. ${ }^{20}$

Sonication time is a significant variable affecting vesicle size $(P=0.001$; Table 3$)$. The use of different sonication times results in different vesicle sizes. Before sonication, large multi-lamellar vesicles with variable size distribution were produced. After ultrasonication, the vesicles were more homogenous, with reduced size because of intramembrane cavitation within the bilayer. Ultrasonic waves travel through

\begin{tabular}{|c|c|c|c|c|}
\hline \multirow[t]{3}{*}{ Factor } & \multicolumn{4}{|l|}{ Response } \\
\hline & \multicolumn{2}{|l|}{$Y_{1}$} & \multicolumn{2}{|l|}{$\underline{Y_{2}}$} \\
\hline & $\begin{array}{l}\text { Estimated } \\
\text { effect }\end{array}$ & $P$-value & $\begin{array}{l}\text { Estimated } \\
\text { effect }\end{array}$ & $P$-value \\
\hline$x_{1}$ & - & $0.044^{*}$ & 33.5 & $0.0083^{*}$ \\
\hline$x_{2}$ & -303.5 & $0.001 *$ & 6.29 & 0.46 \\
\hline$x_{3}$ & -34.75 & 0.47 & -31.09 & $0.011 *$ \\
\hline$x_{1}^{2}$ & -22.833 & 0.74 & 6.46 & 0.60 \\
\hline$x_{1} X_{2}$ & 182.5 & $0.03 I^{*}$ & 2.5 & 0.83 \\
\hline$x_{1} x_{3}$ & 45.0 & 0.507 & -10.5 & 0.39 \\
\hline$x_{2}^{2}$ & 132.66 & 0.099 & 0.04 & 0.99 \\
\hline$x_{2}^{2} x_{3}$ & 30.5 & 0.64 & -5.68 & 0.63 \\
\hline$x_{3}^{2}$ & 95.16 & 0.20 & 13.04 & 0.31 \\
\hline
\end{tabular}

Table 3 Estimated effects and associated $P$-values for all three responses

Note: *Significant effect of factors on individual responses.

Abbreviations: $X_{1}, \%$ L- $\alpha$-phosphatidylcholine to total lipids; $X_{2}$, ultrasonication time (minutes); $X_{3}$, \% ethanol in the hydration medium; $Y_{1}$, mean particle size; $Y_{2}$ vardenafil encapsulation efficiency.

globules and produce compression forces, called cavitation forces, which lead to fractionation of the original globule. These globular fractions transform into long tube-like appendages that can easily be pinched off into small globules. ${ }^{21}$

\section{Entrapment efficiency}

The Pareto chart shows a positive relationship between $X_{1}$ and EE. Increasing amounts of ethanol were found to have a significant negative effect on VRD EE. The interaction of these factors and their quantitative effects on the responses were generated using a mathematical regression equation for the observed response, $Y_{2}$ (Equation 5).

$$
\begin{aligned}
Y_{2}= & 52.7269+0.09175 * X_{1}+3.88525 * X_{2}-1.33642 * X_{3} \\
& +0.00008075 * X_{1}^{2}+0.003125 * X_{1} * X_{2}-0.00105 * X_{1} * X_{2} \\
& +0.005 * X_{1}^{2}-0.0568 * X_{2} * X_{3}+0.010432 * X_{3}^{2}
\end{aligned}
$$

The estimated response surface plots (Figure 2) reveal the relationship between dependent and independent variables. The optimized VRD-NE formulation was prepared using predicted values of $X_{1}, X_{2}$, and $X_{3}$ to measure the observed responses and compare with the calculated values.

The positive relationship between $X_{1}$ and EE can be explained by the emulsification effect of L- $\alpha$ phosphatidylcholine, which decreases interfacial tension between the two phases leading to a homogeneous and stable emulsion. This then enhances VRD entrapment in the NE vesicles. These results are in agreement with data published by Dave et al. Similarly, increasing $\mathrm{CHO}$ concentrations could exclude VRD because of competition between CHO 


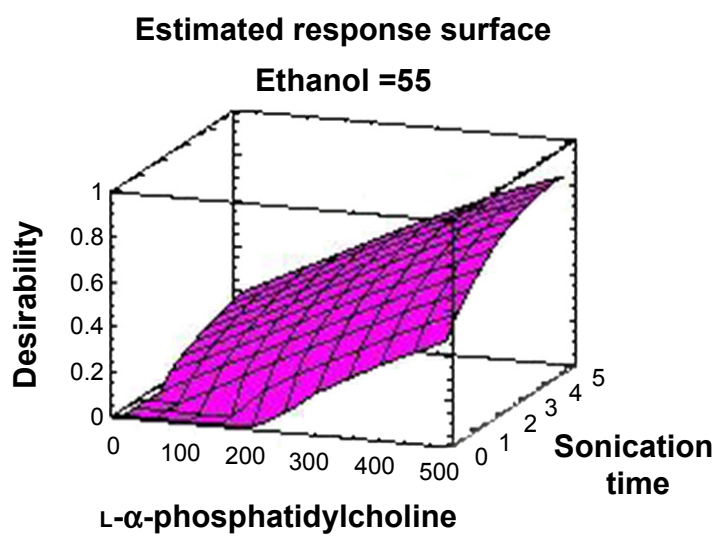

Figure 2 The effects of independent variables and their combined effects on $Y$, and $Y_{2}$ are represented as 3D response surface plots.

Abbreviations: $Y_{1}$, mean particle size; $Y_{2}$, vardenafil encapsulation efficiency.

and VRD for packing space within the vesicular NE bilayer. ${ }^{22}$ According to El-Samaligy et al, increasing the CHO concentration leads to loss of vesicular membrane linearity and disturbs the membrane structure. ${ }^{23}$

Increasing amounts of ethanol were found to have a significant negative effect on VRD EE. This can be attributed to the solubility of both VRD and L- $\alpha$-phosphatidylcholine in ethanol and their insolubility in water. Increased ethanol in the hydration medium will thus solubilize the drug and increase the amount of free VRD in the hydration medium. Sonication time was shown to be a nonsignificant variable $(P=0.46)$. The positive effect of sonication time may be attributed to the enhanced mobility of the surfactant. Rearrangement of lipid molecules also allows for higher entrapment of the drug, with redistribution of the drug inside the NE vesicles. ${ }^{24}$

The observed and predicted values together with residuals for the optimized formulation are shown in Table 4.

\section{VRD-NE vesicle morphology}

The transmission electron microscopy image of the optimized VRD-NE formulation shows a vesicular structure with spherical shape and homogenous unilamellar arrangement (Figure 3). The effect of ethanol on vesicle shape is as observed earlier by Touitou et al. ${ }^{25}$ An ethanol concentration of $20 \%-50 \%$ arranges the ethosomal phospholipid in the form of a tightly packed bilayer with a closed configuration.

Table 4 Predicted and observed values for the optimized VRD-NE

\begin{tabular}{lllll}
\hline Factors & Level & Responses & Predicted & Observed \\
\hline$X_{1}$ & 90 & $Y_{1}$ & 133 & 128 \\
$X_{2}$ & 4.72 & $Y_{2}$ & 78.00 & 76.23 \\
$X_{3}$ & 33.21 & - & - & - \\
\hline
\end{tabular}

Abbreviations: VRD-NE, vardenafil nanoethosomes; $X_{1}, \%$ L- $\alpha$-phosphatidylcholine to total lipids; $X_{2}$, ultrasonication time (minutes); $X_{3}, \%$ ethanol in the hydration medium; $Y_{1}$, mean particle size; $Y_{2}$, vardenafil encapsulation efficiency.

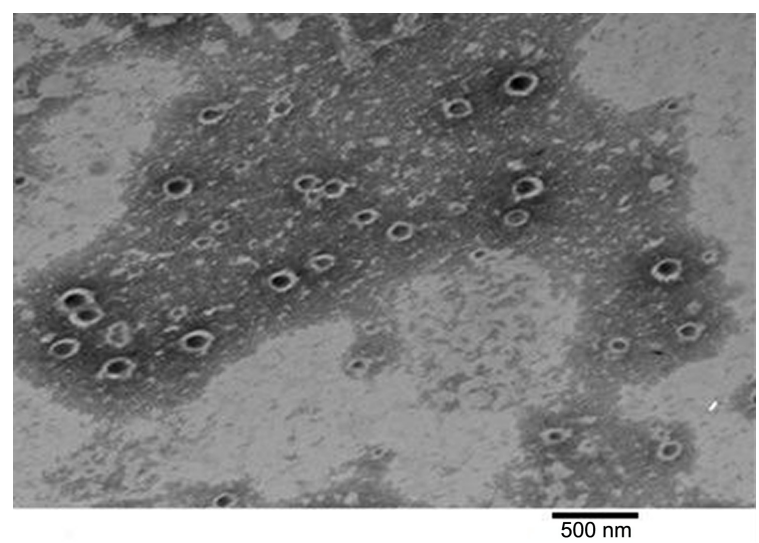

Figure 3 TEM image of optimized VRD-NE.

Abbreviations: TEM, transmission electron microscopy; VRD-NE, vardenafil nanoethosomes.

This can be rationalized by hypothesizing that the motion of the polar head groups of the ethosomal phospholipids is less restricted in the presence of alcohol than in the absence of alcohol. At 50\% ethanol concentration, the signal becomes isotropic and narrow, showing that the phospholipid exists as small, fast-tumbling vesicles. Optimized formula was immediately added to HPMC film solution, so zeta potential (charges which cover the ethosomes) was not considered as an effective parameter or a valuable indicator of stability data in this work.

\section{Drug diffusion}

Comparative permeation profiles of optimized VRD-NE films and unprocessed VRD films are depicted in Figure 4. There was a dramatic rapid diffusion of VRD ( $-30 \%)$ after 1 hour from the optimized NE film; diffusion from the unprocessed drug film did not exceed $4 \%$ after 1 hour. The optimized formulation showed significantly $(P<0.05)$ enhanced

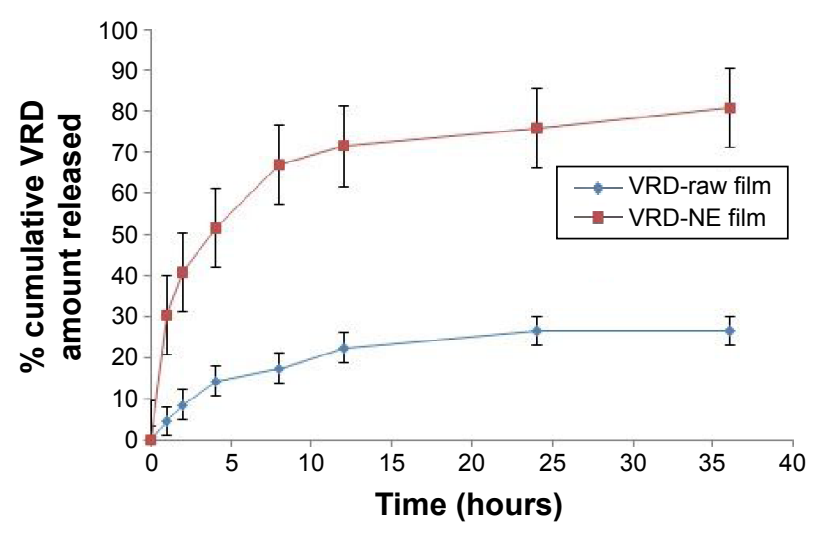

Figure 4 Percentage of cumulative VRD permeation from VRD-NE film and from VRD aqueous suspension through skin.

Abbreviations: VRD, vardenafil; VRD-NE, vardenafil nanoethosomes. 
Table 5 Skin permeation parameters of VRD from nanoethosomal film and VRD raw film through rat abdominal skin

\begin{tabular}{llll}
\hline Parameter & Unit & $\begin{array}{l}\text { VRD raw } \\
\text { film }\end{array}$ & $\begin{array}{l}\text { VRD-NE } \\
\text { formula film }\end{array}$ \\
\hline Steady-state flux & $\mathrm{ng} / \mathrm{cm}^{2} \cdot \mathrm{h}$ & $3.56 \pm \mathrm{I} .32$ & $6.16 \pm 2.12^{*}$ \\
Permeability coefficient & $\mathrm{cm} / \mathrm{h}$ & $0.005 \pm 0.00 \mathrm{I}$ & $0.01 \pm 0.003$ \\
Diffusion coefficient $(D)$ & $\mathrm{cm}^{2} / \mathrm{h}$ & $0.00 \mathrm{I} \pm 0.002$ & $0.004 \pm 0.00 \mathrm{I}^{*}$ \\
Enhancement ratio & - & - & 3.05 \\
\hline
\end{tabular}

Note: $*$ Significant variance $(P$-value $<0.05)$.

Abbreviations: VRD, vardenafil; VRD-NE, vardenafil nanoethosomes.

transdermal flux $\left(6.16 \pm 2.12 \mathrm{ng} / \mathrm{cm}^{2} \cdot \mathrm{h}\right)$ across rat skin compared with unprocessed drug film $\left(3.56 \pm 1.32 \mathrm{ng} / \mathrm{cm}^{2} \cdot \mathrm{h}\right)$. Skin permeation parameters which were calculated from the skin permeation data are shown in Table 5, that are attributed to the effect of ethanol, which acts as penetration enhancer in skin by increasing the fluidity of cell membrane lipids and decreasing the density of cell membrane lipids. ${ }^{26}$ The improved diffusion of the VRD-NE film may also have been facilitated by fusion of ethosomal phospholipids with skin lipids, thereby facilitating VRD-NE permeation into deep skin layers. ${ }^{27}$

\section{CLSM images}

The CLSM study was also conducted to confirm the skin penetration of the vesicles. Top view or $x-y$ plane images of rat skin treated with Rh-labeled HPMC films at varying time periods are presented in Figure 5. Auto fluorescence of the skin (orange) is shown in Figure 5. At 1 hour, HPMC plain film was deposited with a very low intensity at the dermis layer (Figure 5), while VRD-NE were deposited in both the skin surface and the epidermis (Figure 5). Fluorescence intensity from vesicles was high. Because the vesicles were more lipophilic than the plain dye, these vesicles could penetrate into the skin with a higher skin permeation rate than HPMC plain film. At 4 hours, plain HPMC was deposited with a low intensity with traces of orange fluorescence in hypodermis layer, while the orange fluorescence intensity in the case of VRD-NE-HPMC film (Figure 5) was high especially in dermis and hypodermis layers that could be because ethanolic aqueous core could penetrate through the skin.

\section{Bioavailability study}

The AUC for the VRD-NE formulation was $271.67 \mathrm{ng} / \mathrm{mL} \cdot \mathrm{h}$, which was significantly $(P<0.05)$ improved compared with the oral drug suspension $(128.30 \mathrm{ng} / \mathrm{mL} \cdot \mathrm{h})$. A significant $(P<0.05)$ augmentation of $C_{\max }$ was observed with the VRD-NE transdermal film compared with the aqueous suspension. Plasma concentration-time profiles for VRD after transdermal administration of the ethosomal formulation and oral administration of a VRD suspension are shown in Figure 6. Pharmacokinetic parameters are shown in Table 6. The enhanced bioavailability following transdermal administration of the liposomal formulation can be attributed to a combination of factors: firstly, the ethosomal vesicles act as

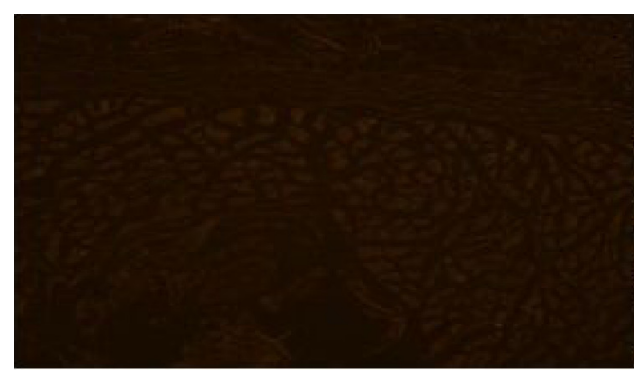

HPMC plain film skin image at 1 hour

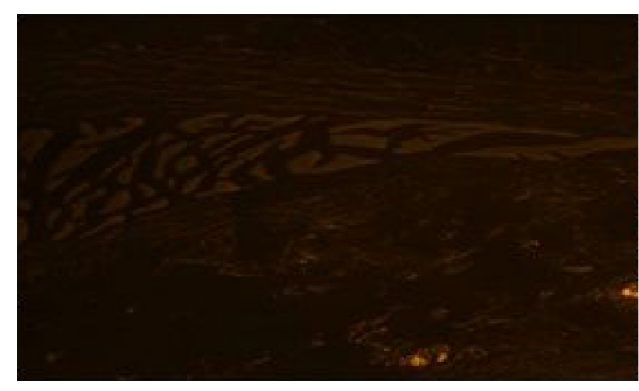

HPMC plain film skin image at 4 hours

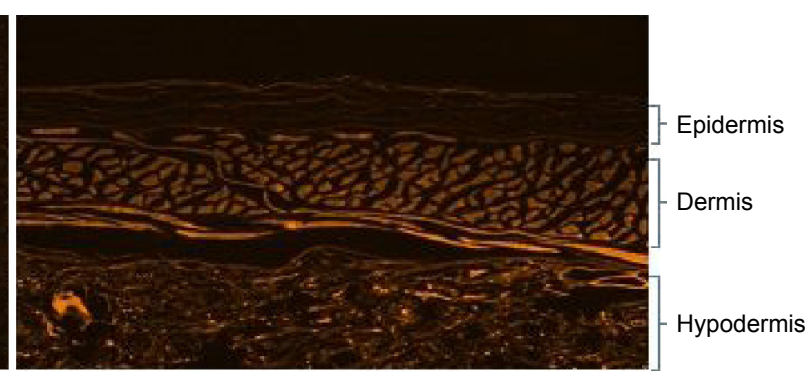

VRD-NE-HPMC film skin image at 1 hour

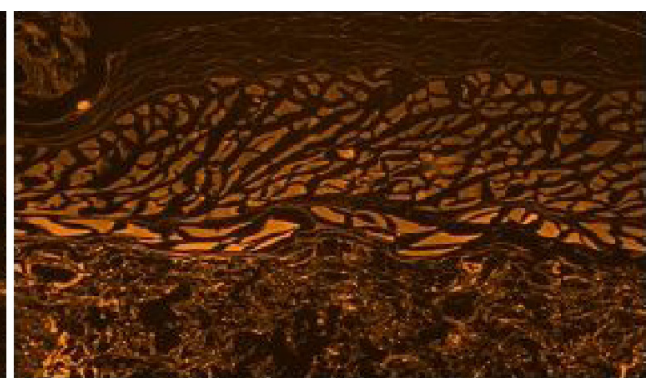

VRD-NE-HPMC film skin image at 4 hours

Figure 5 Confocal laser scanning microscopic images.

Abbreviations: HPMC, hydroxypropyl methylcellulose; VRD-NE-HPMC, vardenafil nanoethosomes with hydroxypropyl methylcellulose. 


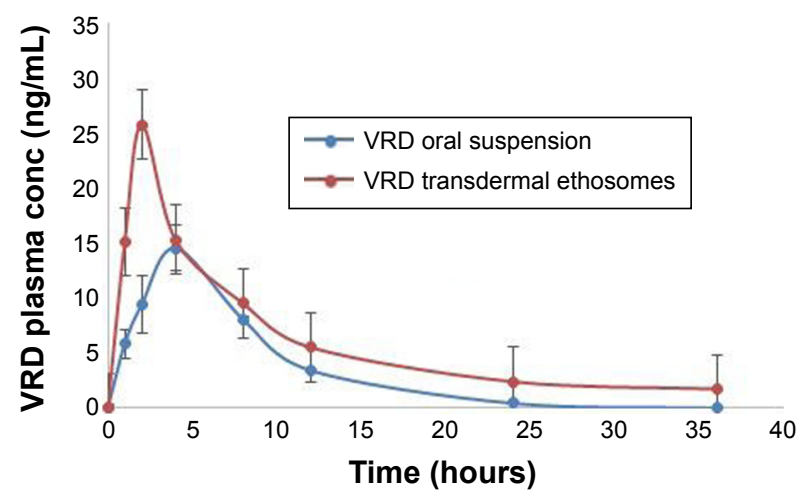

Figure 6 Mean \pm SD plasma concentrations for VRD-NE film and VRD aqueous suspension.

Abbreviations: SD, standard deviation; VRD-NE, vardenafil nanoethosomes; VRD, vardenafil; conc, concentrations.

a delivery vector for VRD to cross skin barriers; secondly, the ethosomal vesicles introduce VRD as a fine dispersion compared with the coarser particles of the VRD oral suspension, and provide an increased surface area with a reduced diffusion path length; and thirdly, the NE vesicles afford a higher adhesion surface contact with the absorption site. The MRT for the VRD-NE film (16.84 \pm 3.54 hours) was higher than that of the VRD oral suspension (7.24 \pm 2.32 hours). This suggests that VRD-NE are retained within the skin layers for longer periods of time and serve as a drug reservoir for extended release into the viable epidermis.

\section{Conclusion}

An optimized nanoethosomal system for transdermal delivery of VRD has been developed to treat ED and evaluated both ex vivo and in vivo. In the preparation of VRD as nanoethosomal vesicles, addition of $\mathrm{CHO}$, and the use of a more rigid phospholipid with an ethanolic aqueous core improved VRD EE within the vesicles. Pharmacokinetic studies in rats showed approximately twofold augmentation of VRD bioavailability from the transdermal films compared with an

Table 6 Pharmacokinetic parameters of VRD-NE after transdermal administration of $2 \mathrm{mg} / \mathrm{kg}$ optimized formula and $2 \mathrm{mg} / \mathrm{kg}$ of VRD aqueous suspension

\begin{tabular}{llll}
\hline Parameter & Unit & $\begin{array}{l}\text { VRD } \\
\text { suspension }\end{array}$ & $\begin{array}{l}\text { VRD-transdermal } \\
\text { nanoethosomes }\end{array}$ \\
\hline$t_{1 / 2}$ & hours & $3.89 \pm 1.23$ & $14.40 \pm 4.54^{*}$ \\
$T_{\max }$ & hours & $4 \pm 10$ & $2 \pm 1.54$ \\
$C_{\max }$ & $\mathrm{ng} / \mathrm{mL}$ & $14.54 \pm 6.32$ & $25.76 \pm 5.39$ \\
$\mathrm{AUC}_{\text {0-infobs }}$ & $\mathrm{ng} / \mathrm{mL} \cdot \mathrm{h}$ & $128.30 \pm 18.87$ & $271.67 \pm 37.43^{*}$ \\
MRT $_{\text {0-inf_obs }}$ & hours & $7.24 \pm 2.32$ & $16.84 \pm 3.54^{*}$ \\
\hline
\end{tabular}

Note: *Significant variance $(P$-value $<0.05)$.

Abbreviations: VRD-NE, vardenafil nanoethosomes; VRD, vardenafil; AUC, area under the plasma concentration-time curve; MRT, mean residency time; $t_{1 / 2}$, half life; $T_{\max }$, time of maximum concentration; $C_{\max }$, maximum concentration. oral drug suspension. VRD-NE transdermal films could thus be used to treat ED, with the potential for lower doses and reduced side effects. VRD-NE transdermal films are a promising transdermal drug delivery system for the treatment of ED that could improve patient tolerability and compliance.

\section{Acknowledgments}

This work was supported by the Deanship of Scientific Research (DSR), King Abdulaziz University, Jeddah (under grant number 166/911/D1435). The author gratefully acknowledges the technical and financial support provided by DSR.

Also, the author would like to express his thanks and appreciation to Dr Osama Abdulhakim, Department of Pharmaceutics \& Industrial Pharmacy, Faculty of Pharmacy, King Abdulaziz University, Dr Khalid El Say, Department of Pharmaceutics \& Industrial Pharmacy, Faculty of Pharmacy, King Abdulaziz University, and Dr Hany El Basossy, Department of Pharmacology, Faculty of Pharmacy, King Abdulaziz University, for their valuable advice during the work.

\section{Disclosure}

The author reports no conflicts of interest in this work.

\section{References}

1. Gratzke C, Angulo J, Chitaley K, et al. Anatomy, physiology, and pathophysiology of erectile dysfunction. J Sex Med. 2010;7(1 Pt 2): 445-475.

2. El-Sakka AI. Erectile dysfunction in Arab countries. Part I: Prevalence and correlates. Arab J Urology. 2012;10(2):97-103.

3. Li J, Shi Q, Pu C, et al. Phosphodiesterase type 5 inhibitors for the treatment of post-nerve sparing radical prostatectomy erectile dysfunction in men. Sci Rep. 2014;4:1-6.

4. Choi MK, Song IS. Characterization of efflux transport of the PDE5 inhibitors, vardenafil and sildenafil. J Pharm Pharmacol. 2012;64(8): 1074-1083.

5. Bruzziches R, Francomano D, Gareri P, Lenzi A, Aversa A. An update on pharmacological treatment of erectile dysfunction with phosphodiesterase type 5 inhibitors. Expert Opin Pharmacother. 2013;14(10): 1333-1344.

6. Weeks JL, Zoraghi R, Beasley A, Sekhar KR, Francis SH, Corbin JD. High biochemical selectivity of tadalafil, sildenafil and vardenafil for human phosphodiesterase 5A1 (PDE5) over PDE11A4 suggests the absence of PDE11A4 cross-reaction in patients. Int J Impot Res. 2004; 17(1):5-9.

7. Doggrell SA. Comparison of clinical trials with sildenafil, vardenafil and tadalafil in erectile dysfunction. Expert Opin Pharmacother. 2005;6(1): 75-84.

8. Park J, Cho W, Kang H, Lee BBJ, Kim TS, Hwang SJ. Effect of operating parameters on $\mathrm{PVP} /$ tadalafil solid dispersions prepared using supercritical anti-solvent process. J Supercrit Fluids. 2014;90:126-133.

9. Schreier H, Bouwstra J. Liposomes and niosomes as a drug carriers: dermal and transdermal drug delivery. J Control Release. 1994;30: $1-15$.

10. Kumazoe M, Tsukamoto S, Lesnick C, et al. Vardenafil, a clinically available phosphodiesterase inhibitor, potentiates the killing effect of EGCG on CLL cells. Br J Haematol. 2015;168(4):610-613. 
11. Touitou E, Dayan N, Bergelson L, Godin B, Eliaz M. Ethosomes novel vesicular carriers for enhanced delivery: characterization and skin penetration properties. J Control Release. 2000;65:403-418.

12. Touitou E, inventor. Composition of applying active substance to or through the skin. United States patent US 5540934. 1996 July 30.

13. Jain S, Tiwary AK, Sapra B, Jain NK. Formulation and evaluation of ethosomes for transdermal delivery of lamivudine. AAPS PharmSciTech. 2007;8(4):249-257.

14. Heinig R, Weimann B, Dietrich H, Böttcher MF. Pharmacokinetics of a new orodispersible tablet formulation of vardenafil. Clin Drug Investig. 2011;31(1):27-41.

15. Maestrelli F, Capasso G, González-Rodríguez ML, Rabasco AM, Ghelardini C, Mura P. Effect of preparation technique on the properties and in vivo efficacy of benzocaine-loaded ethosomes. J Liposome Res. 2009;19(4):253-260.

16. Carlucci G, Palumbo P, Iuliani P, Palumbo G. Development of a method for the determination of vardenafil in human plasma by high performance liquid chromatography with UV detection. Biomed Chromatogr. 2009; 23(7):759-763.

17. Schatzlein A, Cevc G. Non-uniform cellular packing of the stratum corneum and permeability barrier function of intact skin: a high-resolution confocal laser scanning microscopy study using highly deformable vesicles (Transfersomes). Br J Dermatol. 1998;138(4):583-592.

18. De Vries JH, Semeijn C, Buwalda PL, inventors; Coöperatie Avebe U.A., assignee. Emulsifier prepared using a glycosyl transferase. United States patent US 8178323 B2. 2012 May 15.

19. Essa EA. Effect of formulation and processing variables on the particle size of sorbitan monopalmitate niosomes. Asian J Pharm. 2010; 4(4):227.
20. Mokhtar M, Sammour OA, Hammad MA, Megrab NA. Effect of some formulation parameters on flurbiprofen encapsulation and release rates of niosomes prepared from proniosomes. Int J Pharm. 2008;361(1): 104-111.

21. Koch S, Pohl P, Cobet U, Rainov NG. Ultrasound enhancement of liposome-mediated cell transfection is caused by cavitation effects. Ultrasound Med Biol. 2000;26(5):897-903.

22. Dave V, Kumar D, Lewis S, Paliwal S. Ethosome for enhanced transdermal drug delivery of aceclofenac. Int J Drug Deliv. 2011;2(1):81-92.

23. El-Samaligy MS, Afifi NN, Mahmoud EA. Increasing bioavailability of silymarin using a buccal liposomal delivery system: preparation and experimental design investigation. Int J Pharm. 2006;308(1):140-148.

24. Rakesh R, Anoop KR. Formulation and optimization of nano-sized ethosomes for enhanced transdermal delivery of cromolyn sodium. J Pharm Bioallied Sci. 2012;4(4):333.

25. Fahmy UA, Ahmed OA, Hosney KM. Development and evaluation of avanafil self-nanoemulsifying drug delivery system with rapid onset of action and enhanced bioavailability. AAPS PharmSciTech. 2015;16(1):53-58

26. Sinico C, Fadda AM. Vesicular carriers for dermal drug delivery. Expert Opin Drug Deliv. 2009:813-825.

27. Jain S, Jain N, Bhadra D, Tiwary AK, Jain NK. Transdermal delivery of an analgesic agent using elastic liposomes: preparation, characterization and performance evaluation. Curr Drug Deliv. 2005;2(3):223-233.
Drug Design, Development and Therapy

\section{Publish your work in this journal}

Drug Design, Development and Therapy is an international, peerreviewed open-access journal that spans the spectrum of drug design and development through to clinical applications. Clinical outcomes, patient safety, and programs for the development and effective, safe, and sustained use of medicines are a feature of the journal, which

\section{Dovepress}

has also been accepted for indexing on PubMed Central. The manuscript management system is completely online and includes a very quick and fair peer-review system, which is all easy to use. Visit http://www.dovepress.com/testimonials.php to read real quotes from published authors.

Submit your manuscript here: http://www.dovepress.com/drug-design-development-and-therapy-journal 